\title{
A cost-effective high-throughput digital system for observation and acquisition of animal behavioral data
}

\author{
Jonathan A. Farrimond, ANdrew J. Hill, Nicholas A. Jones, \\ Gary J. Stephens, Benjamin J. Whalley, and Claire M. Williams \\ University of Reading, Reading, England
}

\begin{abstract}
We have designed and implemented a low-cost digital system using closed-circuit television cameras coupled to a digital acquisition system for the recording of in vivo behavioral data in rodents and for allowing observation and recording of more than 10 animals simultaneously at a reduced cost, as compared with commercially available solutions. This system has been validated using two experimental rodent models: one involving chemically induced seizures and one assessing appetite and feeding. We present observational results showing comparable or improved levels of accuracy and observer consistency between this new system and traditional methods in these experimental models, discuss advantages of the presented system over conventional analog systems and commercially available digital systems, and propose possible extensions to the system and applications to nonrodent studies.
\end{abstract}

The objective monitoring of animal behavior is necessary in diverse investigations in psychology and in many other disciplines. However, the analysis of data acquired from such monitoring can be labor intensive and time consuming. Such observational analysis typically uses continuous video analysis to determine the frequency, duration, and temporal distribution of various mutually exclusive behavioral categories (e.g., locomotion, eating, drinking, grooming, resting), often via a computerized coding system (e.g., The Observer Video-Pro, Noldus). Furthermore, subsequent offline analysis of recorded behavior is often desirable since it allows accurate analysis of complex, brief, and/or high-frequency behaviors. Offline methods also allow for blinding and analysis of data by observers. Such an approach can increase accuracy and can be used to assess interobserver consistency. Several options for video capture and storage systems are available, although these are frequently inefficient because the use of consumer video cameras often requires laborious manual playback or separate offline encoding of recorded data, or are prohibitively expensive owing to the expense of commercially available complete observation and recording systems $(\sim \$ 10,000)$. High-throughput recording of several animals simultaneously is either inconvenient or expensive.

In our laboratory, researchers investigate drug effects using a variety of voluntary and involuntary behaviors in rodents - for example, feeding (Williams \& Kirkham, 2002; Williams, Rogers, \& Kirkham, 1998) and, more recently, chemically induced seizures. In order to remove the need for analog video recording and avoid incurring the cost of commercial, off-the-shelf observation systems, we have developed a cost-effective system for the monitoring, recording, and subsequent storage of behavioral video data. This system employs closed-circuit television (CCTV) cameras attached via video capture cards to a PC. Public domain camera and video management software is then used to control recording sessions. This system has been validated in two separate experimental models, can record data from as many as 16 cameras even in low light (0.01 lux [lx]), and yields digital video files directly to the PC hard disk for subsequent analysis offline. Additionally, this system is versatile and easy to combine with other equipment to record additional data (e.g., to assess rodent food and water intake; TSE Drinking and Feeding Monitor, TSE, Germany). Thus, the presented system provides a cost-effective, efficient, and reliable tool that is simple to assemble and use for the acquisition and storage of animal behavioral video data. Moreover, the flexibility of output formats supported by this system enables compatibility with a diverse range of offline analysis systems.

\section{METHOD}

\section{Experimental Setup}

All procedures complied with the U.K. Animals (Scientific Procedures) Act, 1986. All data acquired were also used as part of ongoing research projects.

\section{Chemically Induced Seizures}

As part of an investigation studying potential anticonvulsants, adult male $(P>21 ; 60-100 \mathrm{~g})$ Wistar rats were injected intraperitoneally with $80-\mathrm{mg} / \mathrm{kg}$ pentylenetrazol (PTZ; Sigma, U.K.) to induce seizures, and immediately after injection were placed in the 


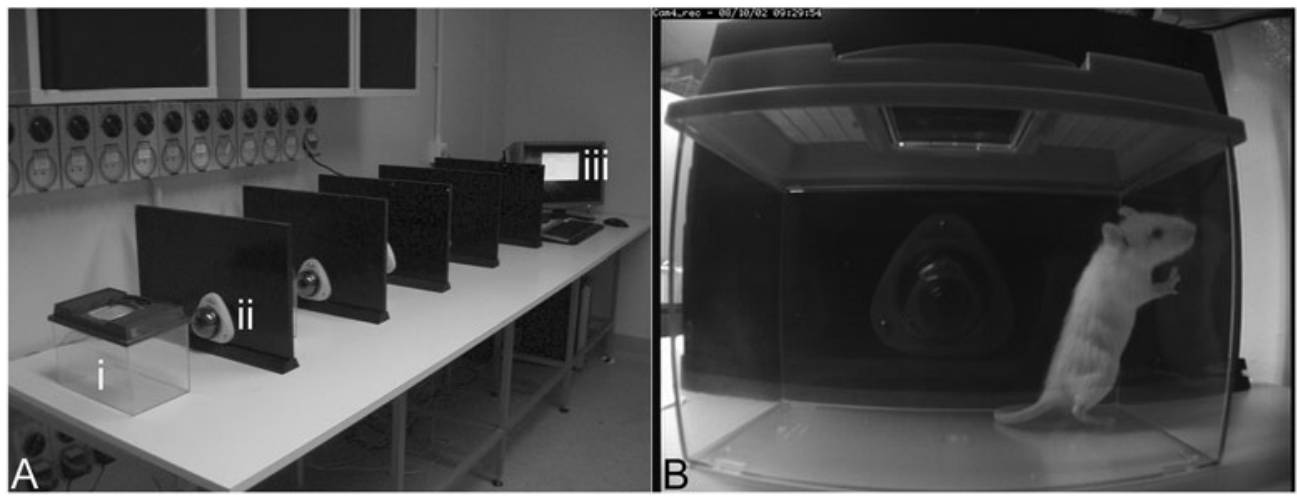

Figure 1. Use of the presented digital observation system in monitoring chemically induced seizures. (A) Experimental setup for monitoring and recording of 5 rats simultaneously, showing (i) the animal tank, (ii) the camera mounted on the divider, and (iii) the PC. (B) Image of rat within monitoring tank prior to seizure induction.

observation tanks. At this point, recording commenced to capture convulsant effects for later observation.

\section{Rodent Feeding}

Food intake levels in 12 male rats $(P>40 ; 200-250 \mathrm{~g}$ on Day 1$)$ were examined simultaneously using a food intake measurement system. A bank of 12 experimental cages containing a barred floor, Perspex rest area, feeding hopper, and water bottles were used. At 10:00 h, animals were weighed and placed in their individual experimental cages, which contained standard lab chow $(\sim 30 \mathrm{~g})$ in computer-weighed feeding hoppers. Food consumption was then monitored, and behavior was observed for $30 \mathrm{~min}$. All feeding and behavioral data were then analyzed offline using The Observer Video-Pro behavioral analysis software.

\section{Analog Acquisition System}

In order to provide a standard against which the new digital system could be compared and validated, we used our former method for acquiring observational data, which employed conventional domestic video recorders (NV-DX110B, Panasonic). In seizure model investigations, a maximum of 3 rats were placed in separate clear tanks (Savic 0129 Fauna Box 6L; Aquatics Online, U.K.) that were $\sim 1 \mathrm{~m}$ away from three domestic portable video cameras, and behavior was recorded to videocassette. For feeding studies, the same cameras were positioned to view the cage from an angle similar to that used by the digital system (see below) at a distance of $\sim 1 \mathrm{~m}$. For both experimental conditions, the recorded analog videotapes were captured in real time to a PC via a USB-205 Audio/Video Grabber (Cypress Technology Co., U.K.) and were digitally encoded to MPEG-2 video. The resulting video file was analyzed using The Observer Video-Pro software.

\section{New Digital Acquisition System}

For observation of chemically induced seizures in rats, five clear tanks (Figure 1A, i) were placed between black wooden dividers with CCTV cameras (TP-101BK, Topica, Taiwan; Figure 1A, ii) mounted on them (distance from tank to camera was $\sim 10 \mathrm{~cm}$ ).

For observation of feeding and behavior, CCTV cameras were positioned above each cage to allow for an unimpeded view (distance from cage to camera was $\sim 10 \mathrm{~cm}$; Figure $2 \mathrm{~A}\left[{ }^{*}\right]$ ). In one instance, in order to allow direct comparison of the analog and digital acquisition systems in feeding and behavioral studies, events in a single cage were recorded simultaneously by both systems. Data were analyzed offline using The Observer Video-Pro software.

\section{Validation of the New Digital Acquisition System}

Following acquisition of behavioral data from the analog system and the digital systems, data were uploaded and coded offline using
The Observer Video-Pro software. Coding was performed twice on each piece of footage to allow an assessment of reliability. Intraobserver reliability analysis, which compares the consistency of scoring between pairs of observers, assessing for both coding and timing errors, generated a statistic (Cohen's kappa) that is an overall measurement of agreement that is corrected for agreements that may occur by chance (Cohen, 1960) and for which a lower value indicates higher consistency.

\section{RESULTS}

\section{Requirements for the System}

We identified several requirements for a new observation system that would replace analog portable video cameras. These requirements included the ability to record simultaneously from many cameras, to allow experiments with as many as 16 animals or permit multiple-angle recordings of fewer animals. The acquired video needed to be in a suitable format and of sufficient quality for accurate offline analysis using The Observer Video-Pro software. The cameras also needed to have short working distances to minimize space usage (as compared with $\sim 1 \mathrm{~m}$ for analog system cameras), to be easily mountable in a number of different ways, and to be functional at low light levels for observations under low- or red-light conditions ( 4 1x). Digital acquisition of data directly to PC hard disk was also a requirement in order to obviate any need for time-consuming real-time conversion of analog video. The system described below fulfilled all of these requirements and, importantly, was cost effective and simple to assemble and use. Figure 3 is a schematic of the digital observation and acquisition system.

\section{Observation System Design}

PC and video equipment. The components for two full systems were obtained. PCs were constructed using 3.16-GHz Intel Core 2 Duo processors with 2-GB RAM and 1-TB hard drives to provide adequate processing speed and disk space during data acquisition. Two monitors (20 in.; Acer, Taiwan) allowed simultaneous viewing of all cameras. To minimize noise that might be disruptive to animals, low-volume fans were fitted to the PCs, and fabric 


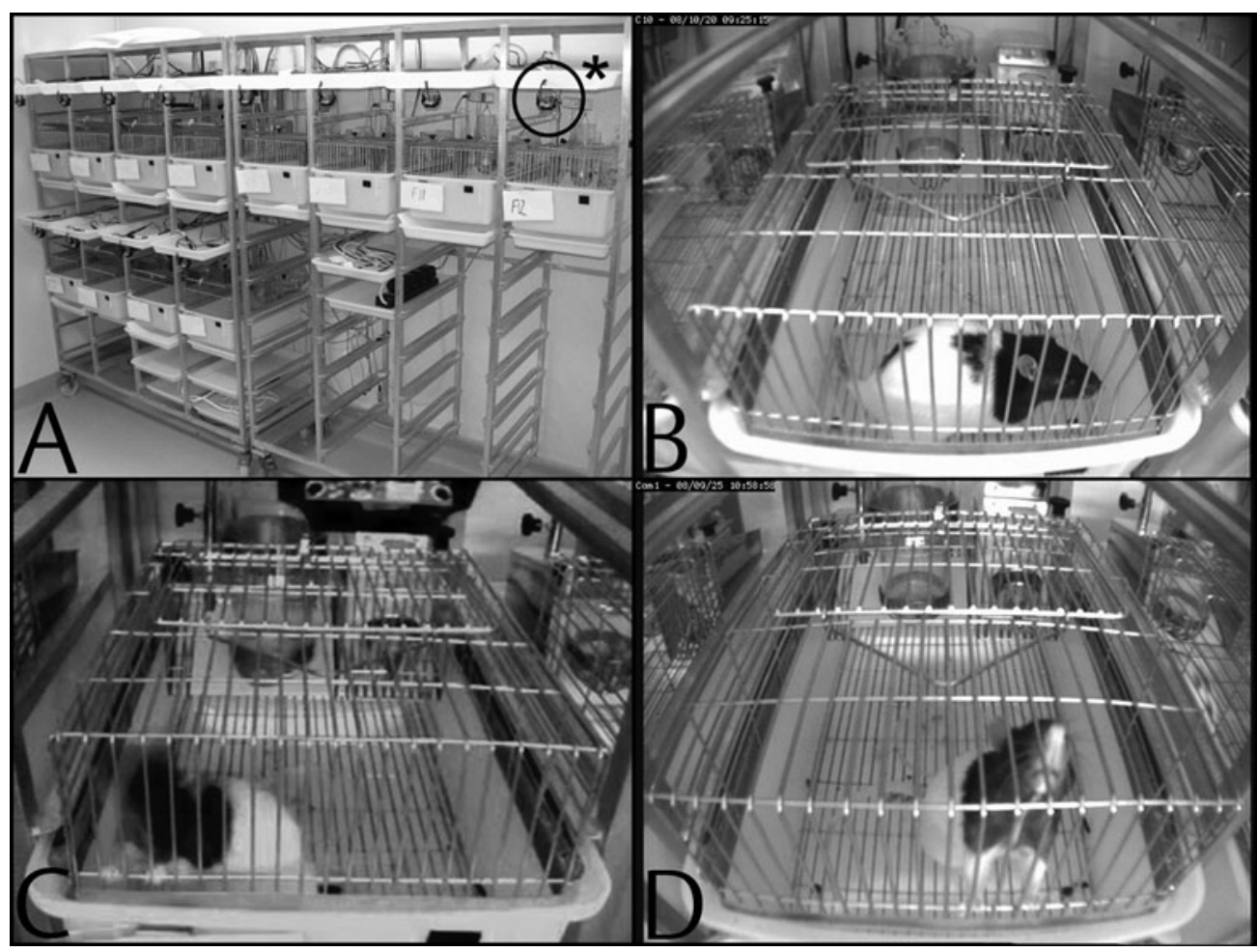

Figure 2. Use of the presented digital observation system for monitoring and recording rodent feeding behavior. (A) Experimental setup and simultaneous monitoring for 12 animals; the asterisk (*) denotes circled example camera. (B) Still frame acquired by using the presented digital observation system of rat in normal light. ( $C$ and $D)$ A comparison of still frames taken by the analog acquisition system (C) and the presented observation system (D) under red-light conditions ( $\sim 4$ lux).

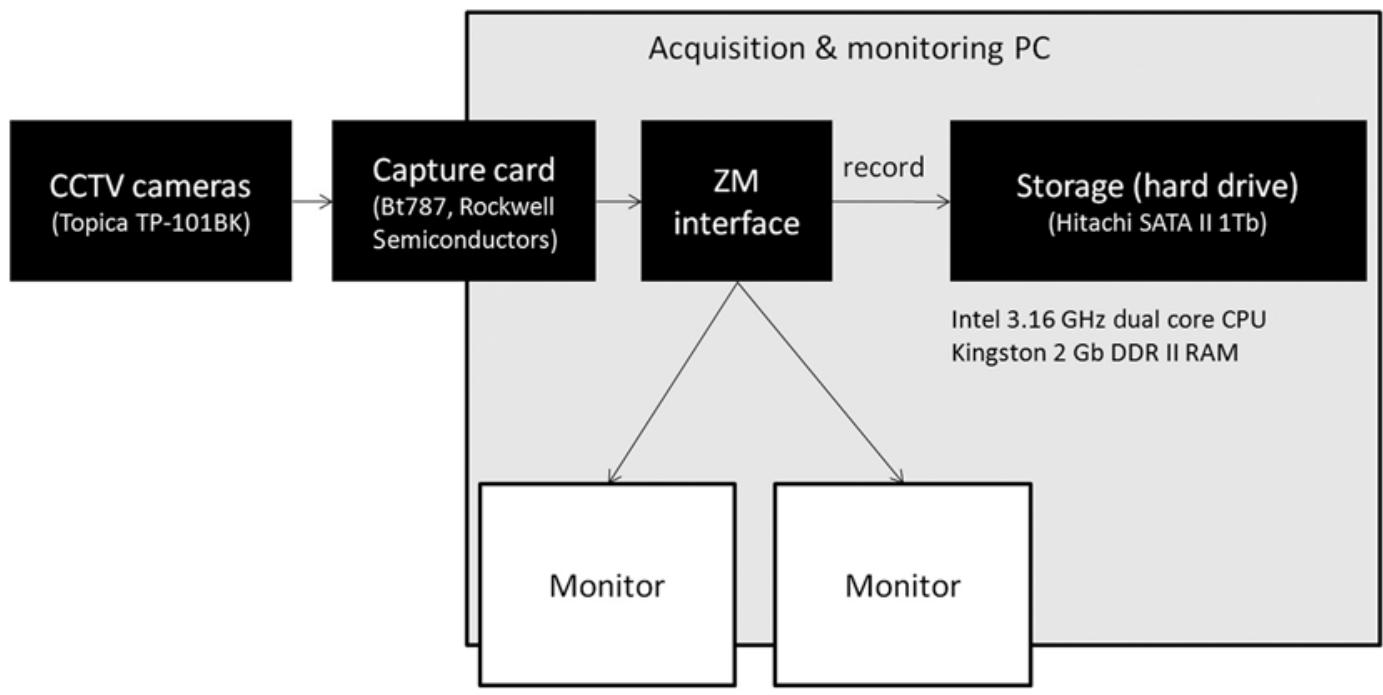

Figure 3. A schematic of the presented digital observation system. Closed-circuit television (CCTV) cameras ( $\leq 16$ ) were connected to an acquisition and monitoring PC via video capture cards for display on dual monitors and simultaneous recording to hard disk. ZoneMinder (ZM) software was used to control the cameras and for monitoring and recording.

gauze was attached to the interior of all computer case vents to limit infiltration of particulate matter. Two 120-framesper-second (FPS) 8-port video capture cards (Bt787, Rockwell Semiconductors, U.S.) were used to connect CCTV cameras to the video acquisition PC. From 5 to 12 Sony
CCD 1/3-in. black-and-white cameras (420TVL [television lines] $0.01 \mathrm{~lx}, 12-\mathrm{V}$ DC) were connected to the computers via BNC cables and were powered via mains (power) adapters. Cameras were distributed equally across the two capture cards to maximize the FPS capture rate. 


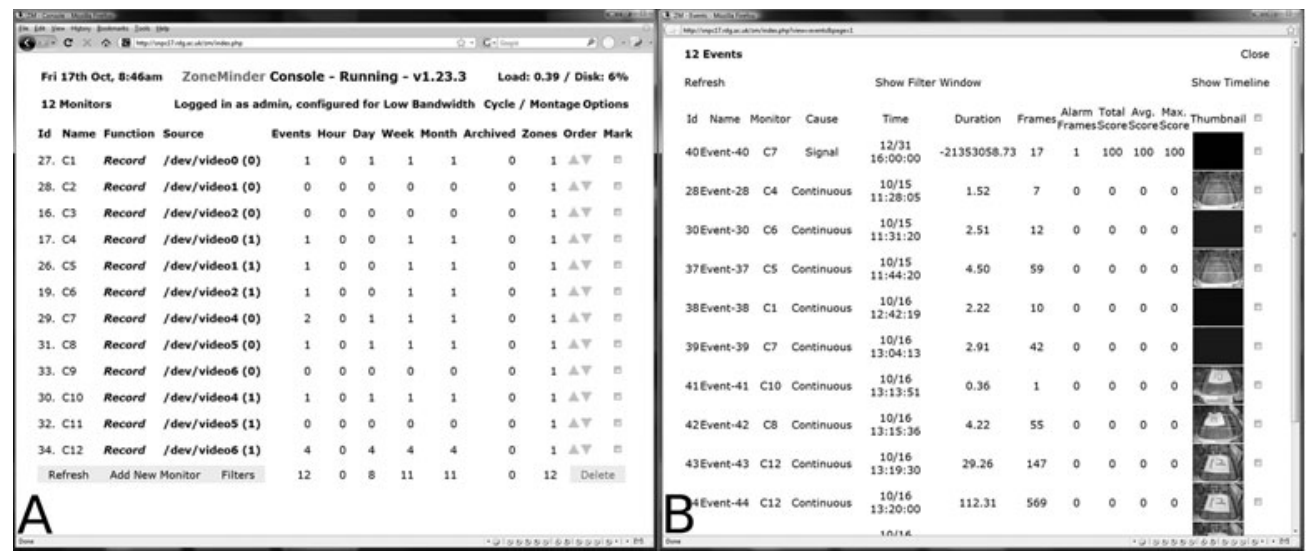

Figure 4. ZoneMinder Web interface. (A) Control panel for 12 cameras. (B) ZoneMinder event log.

PC operating system and software installation. The open source Mandriva Linux distribution operating system software (www.mandriva.com, www.linux.org) was installed on each PC, on the basis of current ease of compatibility with the chosen hardware and acquisition software. However, any Linux-based operating system could be used. The open source ZoneMinder software (www .zoneminder.com, Triornis Ltd, Bristol, U.K.; Figures 4 and 5) was used for video monitoring and acquisition. All software used is available under the GNU Public License (GPL; www.gnu.org/copyleft/gpl.html), allowing free use, distribution, and modification by the user. ZoneMinder provides a Web-based interface to underlying video camera hardware control (Figure 4) running on the open source Apache Web server (www.apache.org), although other Web servers could be used. On entry, this interface provides hashed authentication for local and remote users, thereby securing access to stored data and video observation system control. Such log-in security allows users to monitor ongoing experiments on a separate computer away from the laboratory via a local network or the Internet. Following successful log-in, the user is presented with the principal control screen (Figure 4A), which provides a general overview of connected video devices, a summary of recorded events (a term ZoneMinder uses to describe sections of recorded video; see below), and access to additional settings and controls. Video device control (record or monitor) and review of existing video records are accessed via this interface by clicking on the relevant device or event (Figure 4A). Recording can be started on a device-by-device basis, or a single command can be used to initiate simultaneous recording by groups of devices. Additional settings and controls available via this interface include access, capture, encoding, bandwidth, video montage, and video device cycling, allowing the user to set up and maintain the system through this single Webbased front end. The principal interface also allows Webbased access to all existing video records (Figure 4B), including thumbnail previews. This interface allows events to be replayed (at varying speeds), paused, compressed, securely uploaded, deleted, encoded, archived to alternative servers, and downloaded to a local computer. Batch operations on multiple-event records are also possible via this interface. Acquired video and associated metadata are organized and maintained via a MySQL-based (www .mysql.com) database, with which the end-user requires little, if any, interaction, because the Web-based interface is used to call a number of Perl (www.perl.org) and other (e.g., Bash; www.gnu.org/software/bash) scripts that form part of the ZoneMinder package and perform the necessary administrative and video/image conversion functions. Motion-activated capture, acquisition FPS, continuous recording, and unrecorded monitoring are also accessible via the Web-based interface. The modular and scripted nature of the ZoneMinder system allows experienced users to easily write shell scripts to automate customized, complex tasks from the command line.

Video processing. In order to maintain high capture quality and frame rates from attached cameras, ZoneMinder stores each frame as an incrementally numbered JPEG format image file. Because of limitations to the number of files that can reside in a given directory, long recordings of more that 10,000 images must be broken into events that are stored in separate directories (Figure 4B). After a series of experimental recordings is completed, individual frames within a given event may be converted rapidly to MPEG-2 format video using fastforward MPEG (FFmpeg; ffmpeg.mplayerhq.hu), which can be accessed via the ZoneMinder Web interface. Alternatively, all captured events in a specified range can be converted with the following custom shell script:

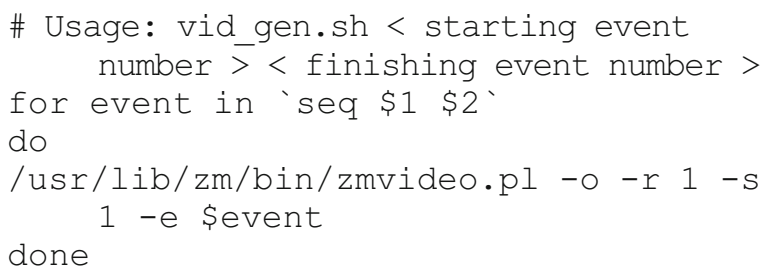

Throughout this process, whether by automated shell script or the ZoneMinder Web interface, the user can specify detailed settings associated with video format $(-f$ and $-F)$, frame rate $(-r)$, size $(-S)$, and scale $(-s)$. Use of the shell script allows the user not to be present during 


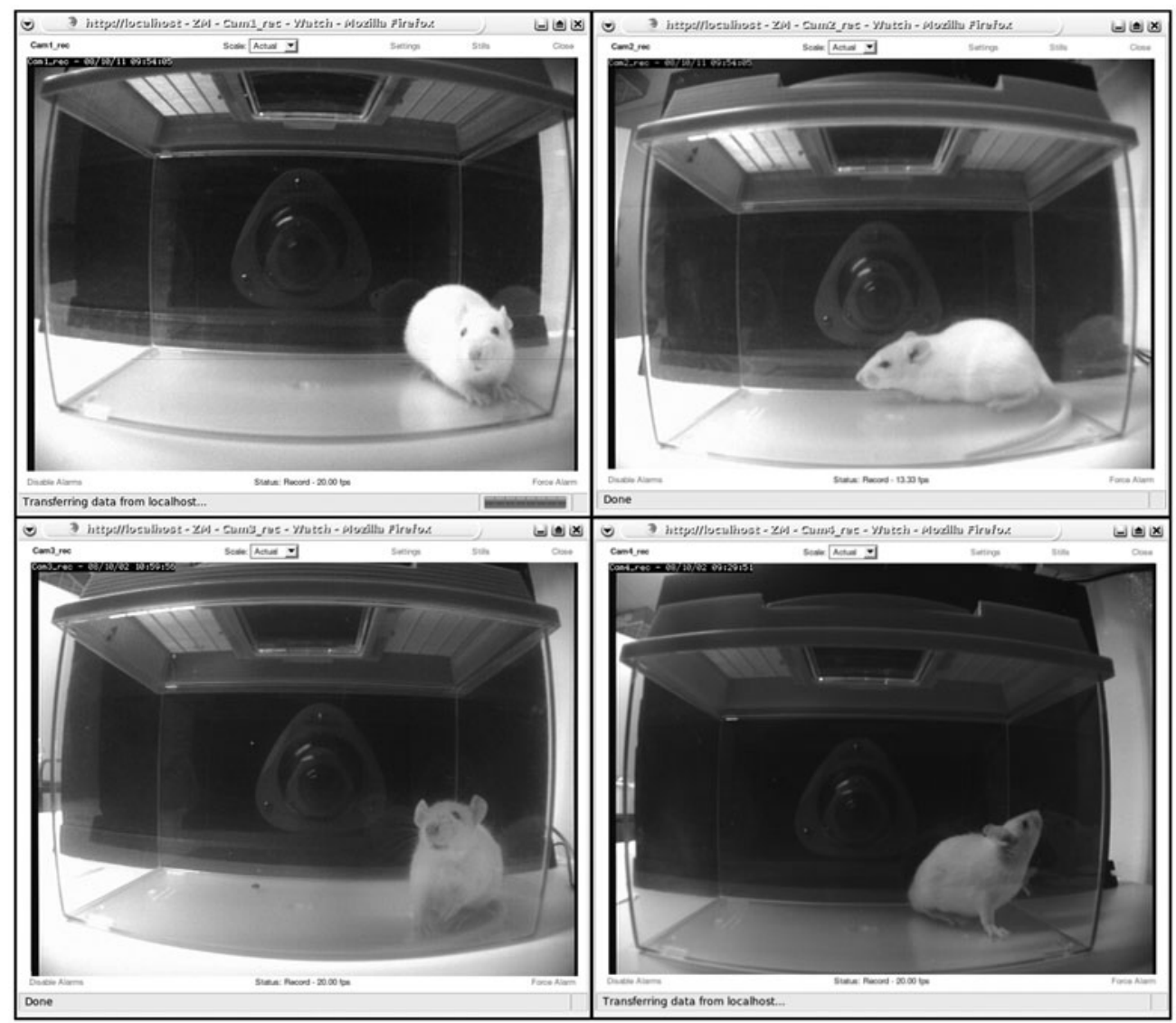

Figure 5. Monitor windows for rats prior to seizure induction acquired simultaneously with the presented digital observation system.

video conversion, which may last several hours if lengthy recordings (which can exceed $15 \mathrm{~h}$ ) must be converted. Thereafter, MPEG-2 encoded event videos may be concatenated manually into a single MPEG file per animal per experiment:

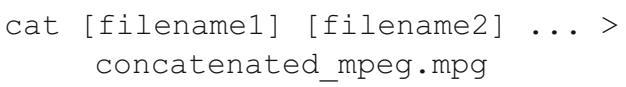

Alternatively, this process may be automated for a series of experiments via a simple shell script and alternative video output used (e.g., Xvid [www.xvid.org] by means of open source encoders such as MEncoder [www.mplayerhq $. h u /])$. Converted and concatenated video files then can be imported directly into The Observer Video-Pro (or alternative coding software) for review and coding.

\section{Use of an Observation System to Record In Vivo Rodent Seizure Activity and Feeding Behavior}

The new observation system was used successfully to record PTZ-induced seizures in as many as 5 rats simultaneously (Figure 5). As described in the Method section, black wooden dividers were used to separate animal tanks as well as serve as camera mountings. To validate the system in this experimental model, the reliability of seizure scoring using The Observer Video-Pro was assessed for both the old analog and new digital systems. Consistency of scoring was comparable using the analog- $(\kappa=.91)$ and digital-acquired video $(\kappa=.92)$. The described system was also used successfully to record a group of 12 rats in individual cages for 7 -h periods and to assess their feeding behavior. To validate the system in this experimental model, identical 30-min pieces of footage from the ana$\log$ and digital systems were coded. Consistency of scoring was improved using the digital-acquired $(\kappa=.94)$, as compared with the analog-acquired $(\kappa=.86)$, video.

\section{DISCUSSION}

The present study describes the successful design, implementation, and validation of a new system for observing and recording in vivo behaviors in rodents. Use of this system has allowed higher throughput recording of greater numbers of animals than was previously possible in our laboratory.

As stated above, the analysis of data acquired from behavioral investigations is highly dependent on the accuracy and reliability of the coding conducted by one or more experimenters. The system presented not only allows for offline analysis of acquired footage, which facilitates blinding and analysis of data by experimenters not necessarily involved with its acquisition, but also has been shown here, by reliability testing, to produce compa- 
rable or indeed increased consistency of scoring of studied behaviors. Factors that increase the consistency and independence of scoring are critical for the production of reliable, high-quality quantitative results in addition to being used to assess interanalyzer consistency.

Moreover, rising costs associated with facilities for conducting behavioral investigations with animals, coupled with the increased pressure for available space to be used efficiently, are also addressed by the presented system. Because of the low noise and networked nature of the presented acquisition system, it can be situated in the same room as that in which the animals are housed (Figure 1A, iii), and the short working distances of the CCTV cameras (which can be modified) allow a compact experimental area, in comparison with the area required to use an equivalent number of domestic portable cameras. Such a setup permits either direct control of the acquisition system in investigations where the experimenter must be present at all times (e.g., seizure models) or matching levels of control from a remote location when the presence of the experimenter would disrupt the subjects (e.g., feeding studies). Secure remote viewing features also permit intermittent observation of experiments by other researchers, eliminating the need for potentially disruptive staff movements.

A complete system costs $\sim \$ 1,700$, excluding tax. This specification includes 12 cameras, all necessary connectors and cables, capture cards, and the data-acquisition PC. To achieve the same result using domestic portable analog video cameras would cost $\sim \$ 2,360-\$ 15,100$ (depending on quality and functionality and excluding tax). Furthermore, the necessity of laborious encoding of analog-to-digital video files in real time would remain. Alternatively, commercially available systems that provide similar, albeit more limited, functionality as the presented system have significantly higher purchase costs $(\sim 9,450)$. The specified components of such commercial systems are comparable to or of a lower quality than those used to construct the presented system. Thus, the raised cost of commercial systems likely rests with the costs associated with proprietary control software and product support. Our use of freely available, open source software has eliminated software costs, yet this software is still easily integrated with tools that are widely used and robustly tested. Moreover, support for the open source operating system distribution and software is widely available, which often is considered to be one of the open source software's most notable strengths. This lower cost of ownership also encourages the testing and implemen- tation of extensions to the described system for custom applications through the use of additional open source packages. Such extensions include batch automation of video conversion, automated data backups, webcasting of data, image recognition, and motion-sensitive audio and area capture recording.

We believe that the presented system aims to offer an easy-to-use and cost-effective digital video observation solution. However, it should be noted that, as with any in-house system, careful consideration should be given to hardware specification prior to ordering and assembly of components, because the limitations of a system are determined largely by the specific uses to which it may be put. Particular considerations should include disk space and speed requirements, maximum number of cameras per capture card, and overall processing power of the PC. Additionally, organization of overnight batch encoding of acquired video footage is required, because the processing overheads associated with encoding prevent simultaneous capture and encoding.

Beyond the observation and recording of rodent behavior, we would expect that this system, with or without the extensions described above, would be well suited to other behavioral investigations that require unobtrusive recording from several angles (e.g., preferential looking paradigms, focus group studies, and parent-child interaction). In conclusion, we believe that the presented system provides a cost-effective and highly functional solution to many of the challenges presented by behavioral studies.

\section{AUTHOR NOTE}

This research was supported in part by the University of Reading Research Endowment Trust Fund (to C.M.W. and B.J.W.). The authors thank Trevor Jenkinson and his team for technical assistance. Address correspondence to A. J. Hill, School of Psychology and Clinical Language Sciences, University of Reading, Earley Gate, Whiteknights, Reading RG6 6AL, England (e-mail: a.j.hill@reading.ac.uk).

\section{REFERENCES}

CoHEn, J. (1960). A coefficient of agreement for nominal scales. Educational \& Psychological Measurement, 20, 3746.

Williams, C. M., \& KiRKhaM, T. C. (2002). Observational analysis of feeding induced by $\Delta^{9}$-THC and anadamide. Physiology \& Behavior, 76, 241-250.

Williams, C. M., Rogers, P. J., \& KirKham, T. C. (1998). Hyperphagia in pre-fed rats following oral $\Delta^{9}$-THC. Physiology \& Behavior, 65, 343-346.

(Manuscript received October 28, 2008; revision accepted for publication February 19, 2009.) 